\section{Electronically tunable phase response for phased array patches}

\section{P. Padilla, J.M. Fernández-González, A. Muñoz-Acevedo,} J.L. Padilla and M. Sierra-Castañer

The design and prototyping of radiating elements with tunable phase response, for reconfigurable patch array antennas, is presented. Different options are found in the technical literature to obtain feasible reconfigurable array antennas: placing the active circuitry in the transmission lines or directly over the radiating element, obtaining the desired phase shift for each radiating element of the array. In this reported work, the second option is selected and active radiating elements at $12 \mathrm{GHz}$ are designed and prototyped.

Introduction: Phased array antennas are widely used to overcome strict radiation pattern requirements, since actual communication systems are more and more demanding. Phased array configurations enable obtaining different patterns depending on the transmission phase and amplitude of the radiated signal of each array radiating element $[1,2]$. Electronically variable phase behaviour at each array element is directly translated into phase array variation and pattern modifications. In this Letter, active circuitry is integrated directly over each radiating element (stacked patch element), in order to electronically reconfigure the phase response of the radiating element in terms of transmission. These devices are designed for their application in phased array devices such as reflectarrays [3] or artificial lenses [4].

Reconfigurable patches: Every radiating element could be analysed as an equivalent circuit with particular equivalent input impedance. In the literature, tunable lumped elements (pin diodes, varactors, MEMs) are typically used in the electric configuration of a radiating element to vary the working frequency of the antenna, maintaining a required matching level for this frequency [5]. However, there are applications in which the importance does not remain in frequency tuning but in transmission phase response tuning, for a fixed working frequency [6] In this case (multilayered patch structure), modifying the patch equivalent impedance, it is possible to change the working frequency of the patch and, as a consequence, its behaviour in terms of S-parameters. For the current application, variation of the $\arg \left(\mathrm{S}_{21}\right)$ of the structure is desired. Regarding $\left|\mathrm{S}_{11}\right|$ behaviour and matching requirements, a level lower than $-10 \mathrm{~dB}$ is maintained at the working frequency, in order to obtain a wide $S_{21}$ phase variation range.
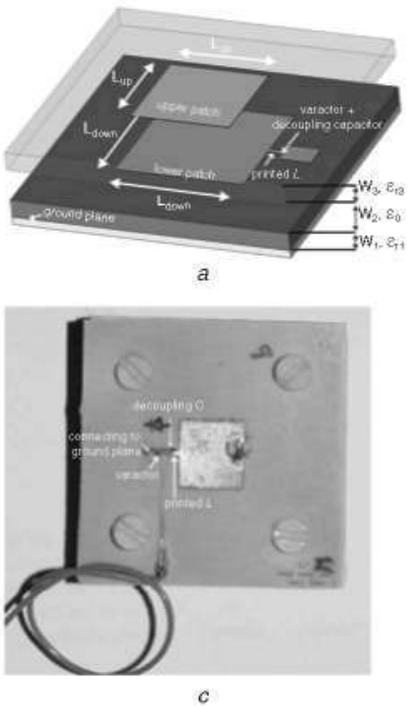
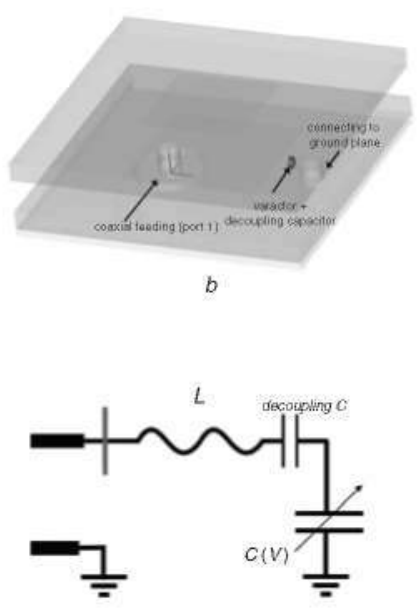

d
Fig. 1 Model for stacked patch radiating element with reconfigurable phase response

$a$ Detailed model with dimensions of structure

$b$ Prototype detail of lower patch with varactor

$c$ Details of feeding port connection (port 1)

$d$ Active LC circuit schematic

Design: To add reconfigurability to the radiating element, an active circuit (LC circuit) is connected to it. A microwave varactor is the element that provides a capacitance variation in the active circuit and, by means of this variation, small changes in the radiating element working frequency $(12 \mathrm{GHz})$ and, what is the main contribution of this Letter, phase response variation. Regarding the inductance in the LC circuit, the needed inductance value is replaced by an equivalent printed circuit [6]. Figs. $1 a$ and $b$ show the selected design model, which has been widely studied with electromagnetic simulation software (CST Microwave Studio 2009), where a radiating horn (port 2) is the excitation probe that illuminates the structure (port 1) with normal incidence. Fig. 2 shows $\arg \left(S_{21}\right)$ and $\left|S_{11}\right|$ simulation results for different capacitance values of the selected varactor (M/A-COM MA46585).
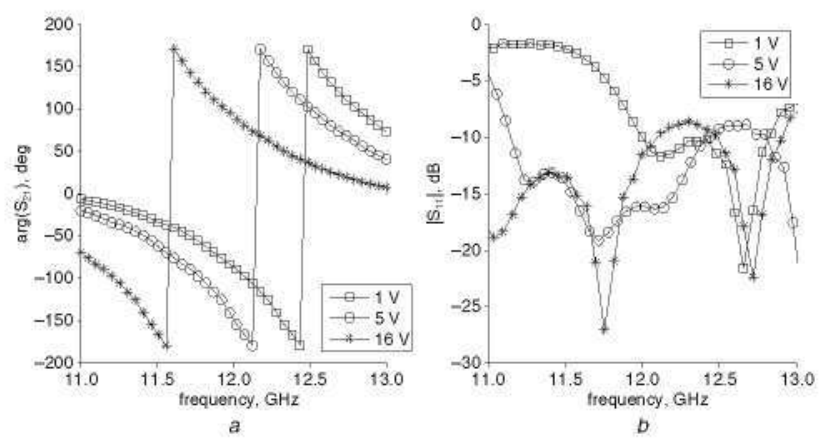

Fig. 2 Simulation results for different capacitance values $a \arg \left(\mathrm{S}_{21}\right)$ $b\left|\mathrm{~S}_{11}\right|$

Prototypes and results: According to the simulated design parameters, prototypes were manufactured. The varactor mounting constrains to avoid damage force a low soldering temperature: epoxy soldering techniques are chosen to overcome these constraints. Fig. $1 b$ shows one of the built prototypes along with some details. The active stacked patch structure itself consisted of two patches on a rectangular lattice with dimensions (Fig. 1c): $\mathrm{L}_{\text {up }} 6.2 \mathrm{~mm}, \mathrm{~L}_{\text {down }} 8.1 \mathrm{~mm}, \mathrm{~W}_{1} 1.575 \mathrm{~mm}, \mathrm{~W}_{2}$ $2 \mathrm{~mm}, \mathrm{~W}_{3} 1.575 \mathrm{~mm}, \epsilon_{\mathrm{r} 1} 2.17, \epsilon_{\mathrm{r} 2} 2.17$. Varactor diodes with a tunable capacitance range from $\sim 0.12 \mathrm{pF}(16 \mathrm{~V})$ to $\sim 2 \mathrm{pF}(0 \mathrm{~V})$ were chosen.

Table 1: $\arg \left(\mathrm{S}_{21}\right)$ variation at $12 \mathrm{GHz}$ against control voltage (values referred to $0 \mathrm{~V}$ )

\begin{tabular}{|c|c|c|}
\hline Voltage $(\mathrm{V})$ & Simulated $\arg \left(\mathrm{S}_{21}\right)(\mathrm{deg})$ & Measured $\arg \left(\mathrm{S}_{21}\right)(\mathrm{deg})$ \\
\hline 0 & 0 & 0 \\
\hline 2 & -16 & -14 \\
\hline 4 & -36 & -42 \\
\hline 6 & -65 & -72 \\
\hline 8 & -90 & -82 \\
\hline 10 & -120 & -110 \\
\hline 12 & -138 & -134 \\
\hline 14 & -160 & -154 \\
\hline 16 & -183 & -176 \\
\hline
\end{tabular}
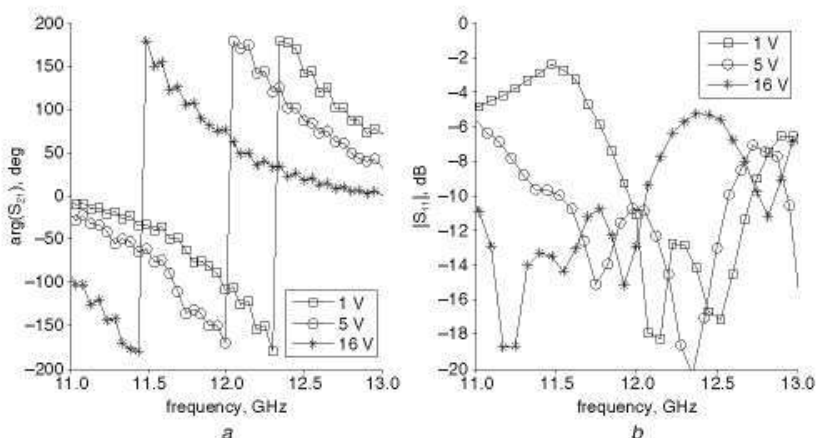

a

Fig. 3 Measurement results for different voltage values $a \arg \left(\mathrm{S}_{21}\right)$ $b\left|\mathrm{~S}_{11}\right|$

The measurements of $\arg \left(\mathrm{S}_{21}\right)$ are obtained with a horn probe (port 2) that illuminates the prototype (port 1) with normal incidence, at a 
distance $d$ much greater than the wavelength at $12 \mathrm{GHz}\left(\lambda_{0}\right)$. Measured $\left|\mathrm{S}_{11}\right|$ and $\arg \left(\mathrm{S}_{21}\right)$ are provided in Fig. 3, for different control voltages (in Fig. $3 a$ the phase component owing to distance $d$ has been subtracted from measurements). According to linearity, a quite linear phase/ voltage relation is obtained (enough for phased array or transmit-array lens application [6]), as it is observed in Table 1.

This device is of interest for microwave systems, such as phased array antennas, and is being applied in the development of electronically reconfigurable transmit-array lenses for space application. In this last case, each lens cell is composed of two active patches and a complete $360^{\circ} \arg \left(\mathrm{S}_{21}\right)$ variation can be achieved.

Conclusions: Presented is the complete description of an electronically reconfigurable radiating element in terms of transmission phase response. The designed device provides a continuous phase variation up to $180^{\circ}$, which is useful for array lens structures. Some design criteria, simulation results and prototype measurements have been presented

Acknowledgments: The device simulations have been carried out using CST Microwave Studio 2009 under cooperative agreement between CST and the Technical University of Madrid. NY substrate used in the prototypes was kindly provided by NELTEC S.A.
J.M. Fernández-González, A. Muñoz-Acevedo and M. Sierra-Castañer (Department of Signal Systems and Radiocommunications, Technical University of Madrid, Spain)

J.L. Padilla (Departamento Electrónica y Tecnología de Computadores, Universidad de Granada, Spain)

\section{References}

1 Padilla, P., and Sierra-Castañer, M.: 'Design and prototype of a $12 \mathrm{GHz}$ transmit-array', Microw. Opt. Technol. Lett., 2007, 49, (12), pp. $3020-3026$

2 Popovic, Z., and Mortazawi, A.: 'Quasi-optical transmit/receive front end', IEEE Trans. Microw. Theory Tech., 1998, 46, (11), pp. 1964-1975

3 Encinar, J.A., and Zornoza, J.: 'Broadband design of three-layer printed reflectarrays', IEEE Trans. Antennas Propag., 2001, 49, (10), pp. $1662-1664$

4 Padilla, P., Muñoz-Acevedo, A., and Sierra-Castañer, M.: 'Passive planar transmit-array microstrip lens for microwave purpose', Microw. Opt. Technol. Lett., 2010, 52, (4), pp. 940-946

5 Haskins, P.M., et al.: 'Active patch antenna element with diode tuning', Electron. Lett., 26, 1991

6 Padilla, P., Muñoz-Acevedo, A., Sierra-Castañer, M., and Sierra-Pérez, M.: 'Electronically reconfigurable transmitarray at $\mathrm{Ku}$ band for microwave applications', IEEE Trans. Antennas Propag., 2010, 58, (8), pp. $2571-2579$ 